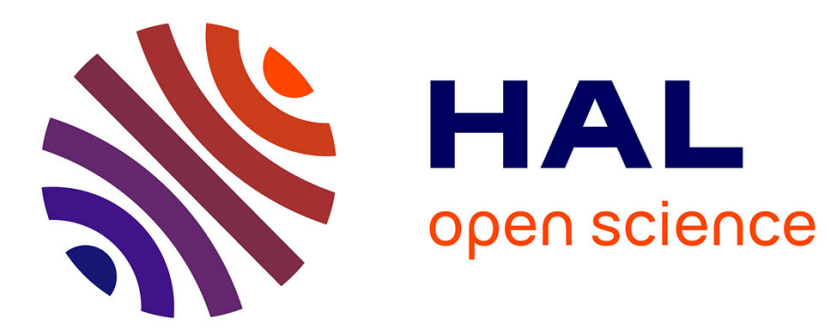

\title{
Random dynamical response of a multibody system with uncertain rigid bodies
}

\author{
Anas Batou, Christian Soize
}

\section{To cite this version:}

Anas Batou, Christian Soize. Random dynamical response of a multibody system with uncertain rigid bodies. COMPDYN 2011, ECCOMAS Thematic Conference on Computational methods in structural dynamics and earthquake engineering, May 2011, Corfu, Greece. pp.1-11. hal-00693071

\section{HAL Id: hal-00693071 \\ https://hal.science/hal-00693071}

Submitted on 1 May 2012

HAL is a multi-disciplinary open access archive for the deposit and dissemination of scientific research documents, whether they are published or not. The documents may come from teaching and research institutions in France or abroad, or from public or private research centers.
L'archive ouverte pluridisciplinaire HAL, est destinée au dépôt et à la diffusion de documents scientifiques de niveau recherche, publiés ou non, émanant des établissements d'enseignement et de recherche français ou étrangers, des laboratoires publics ou privés. 


\title{
RANDOM DYNAMICAL RESPONSE OF A MULTIBODY SYSTEM WITH UNCERTAIN RIGID BODIES
}

\author{
A. Batou ${ }^{1}$, C. Soize ${ }^{1}$, \\ ${ }^{1}$ Université Paris-Est, Laboratoire Modélisation et Simulation Multi Echelle, MSME UMR 8208 \\ CNRS, \\ 5 bd Descartes, 77454 Marne-la-Vallee, France \\ e-mail: \{anas.batou,christian.soize\}@univ-paris-est.fr
}

Keywords: Uncertain rigid body, Random mass, Random center of mass, Random tensor of inertia.

\begin{abstract}
This research is devoted to the construction of the random dynamical response of a multibody system with uncertain rigid bodies. We first construct a stochastic model of an uncertain rigid body by replacing the mass, the center of mass and the tensor of inertia by random variables. The prior probability distributions of the stochastic model are constructed using the maximum entropy principle under the constraints defined by the available information. The generator of independent realizations corresponding to the prior probability distribution of these random quantities are developed. Then, several uncertain rigid bodies can be linked each others in order to calculate the random response of a multibody dynamical system. An application is proposed to illustrate the theoretical development.
\end{abstract}




\section{INTRODUCTION}

This study is devoted to the construction of a probabilistic model of uncertainties for a rigid multibody dynamical system made up of uncertain rigid bodies. In some cases, the mass distribution inside a rigid body is not perfectly known and must be considered as random (for example, the distribution of passengers inside a vehicle) and therefore, this unknown mass distribution inside the rigid body induces uncertainties in the model of this rigid body. Here, we propose a new probabilistic modeling for uncertain rigid bodies in the context of the multibody dynamics. Concerning the modeling of uncertainties in multibody dynamical system, a very few previous researches have been carried out. These researches concerned parameters which describe the joints linking each rigid body to the others and the external sources (see [7], [16], [3]), [12], [13]), but not rigid bodies themselves. In the field of uncertain rigid bodies, a first work has been proposed in [9, 10], in which the authors take into account uncertain rigid bodies for rotor dynamical systems using the nonparametric probabilistic approach [18, 19] consisting in replacing the mass and gyroscopic matrices by random matrices.

In this paper, a general and complete stochastic model is constructed for an uncertain rigid body. The mass, the center of mass and the tensor of inertia which describe the rigid body are modeled by random variables. The prior probability distributions of the random variables are constructed using the maximum entropy principle [6] from Information Theory [17]. The generator of independent realizations corresponding to the prior probability distributions of these random quantities are developed and presented. Then, several uncertain rigid bodies can be linked each others in order to calculate the random response of an uncertain multibody dynamical system. The stochastic multibody dynamical equations are solved using the Monte Carlo simulation method.

Section 2 is devoted to the construction of the mean model for the rigid multibody dynamical system by using the classical method. In Section 3 , firstly, we propose a general probability model for an unconstrained uncertain rigid body and secondly, the uncertain rigid multibody dynamical system is obtained by joining this unconstrained uncertain rigid body to the other rigid bodies. The last section is devoted to an application which illustrates the proposed theory.

\section{MEAN MODEL FOR THE RIGID MULTIBODY DYNAMICAL SYSTEM}

In this paper, the usual model of a rigid multibody dynamical system for which all the mechanical properties are known will be called the mean model (or the nominal model). This section is devoted to the construction of the mean model for a rigid multibody dynamical system. This mean model is constructed as in $([14,15])$ and is summarized below.

\subsection{Dynamical equations for a rigid body of the multibody system}

Let $\mathrm{RB}_{i}$ be the rigid body occupying a bounded domain $\Omega_{i}$ with a given geometry. Let $\xi$ be the generic point of the three dimensional space. Let $\mathbf{x}=\left(x_{1}, x_{2}, x_{3}\right)$ be the position vector of point $\xi$ defined in a fixed inertial frame $\left(O, x_{0,1}, x_{0,2}, x_{0,3}\right)$, such that $\mathbf{x}=\overrightarrow{O \xi}$. The rigid body class is then defined by three quantities.

(1) The first one is the mass $m_{i}$ of $\mathrm{RB}_{i}$ which is such that

$$
m_{i}=\int_{\Omega_{i}} \rho(\mathbf{x}) d \mathbf{x}
$$

where $\rho(\mathbf{x})$ is the mass density. 
(2) The second quantity is the position vector $\mathbf{r}_{i}$ of the center of mass $G_{i}$, defined in the fixed inertial frame, by

$$
\mathbf{r}_{i}=\frac{1}{m_{i}} \int_{\Omega_{i}} \mathbf{x} \rho(\mathbf{x}) d \mathbf{x}
$$

(3) Let $\left(G_{i}, x_{i, 1}^{\prime}, x_{i, 2}^{\prime}, x_{i, 3}^{\prime}\right)$ be the local frame for which the origin is $G_{i}$ and which is deduced from the fixed frame $\left(O, x_{0,1}, x_{0,2}, x_{0,3}\right)$ by the translation $\overrightarrow{O G_{i}}$ and a rotation defined by the three Euler angles $\alpha_{i}, \beta_{i}$ and $\gamma_{i}$. The third quantity is the positive-definite matrix $\left[J_{i}\right]$ of the tensor of inertia in the local frame such that

$$
\left[J_{i}\right] \mathbf{u}=-\int_{\Omega_{i}} \mathbf{x}^{\prime} \times \mathbf{x}^{\prime} \times \mathbf{u} \rho\left(\mathbf{x}^{\prime}\right) d \mathbf{x}^{\prime} \quad, \quad \forall \mathbf{u} \in \mathbb{R}^{3},
$$

in which the vector $\mathbf{x}^{\prime}=\left(x_{1}^{\prime}, x_{2}^{\prime}, x_{3}^{\prime}\right)$ of the components of vector $\overrightarrow{G_{i} \xi}$ are given in $\left(G_{i}, x_{i, 1}^{\prime}, x_{i, 2}^{\prime}, x_{i, 3}^{\prime}\right)$. In the above equation, $\mathbf{u} \times \mathbf{v}$ denotes the cross product between the vectors $\mathbf{u}$ and $\mathbf{v}$.

\subsection{Matrix model for the rigid multibody dynamical system}

The rigid multibody dynamical system is made up of $n_{b}$ rigid bodies and ideal joints including rigid joints, joints with given motion (rheonomic constraints) and vanishing joints (free motion). The interactions between the rigid bodies are realized by these ideal joints but also by springs, dampers or actuators which produce forces between the bodies. In this paper, only $n_{c}$ holonomic constraints are considered. Let $\mathbf{u}$ be the vector in $\mathbb{R}^{6 n_{b}}$ such that $\mathbf{u}=\left(\mathbf{r}_{1}, \ldots, \mathbf{r}_{n_{b}}, \mathbf{s}_{1}, \ldots, \mathbf{s}_{n_{b}}\right)$ in which $\mathbf{s}_{i}=\left(\alpha_{i}, \beta_{i}, \gamma_{i}\right)$ is the rotation vector. The $n_{c}$ constraints are given by $n_{c}$ implicit equations which are globally written as $\boldsymbol{\varphi}(\mathbf{u}, t)=0$. The $\left(6 n_{b} \times 6 n_{b}\right)$ mass matrix $[M]$ is defined by

$$
[M]=\left[\begin{array}{cc}
{\left[M^{r}\right]} & 0 \\
0 & {\left[M^{s}\right]}
\end{array}\right],
$$

where the $\left(3 n_{b} \times 3 n_{b}\right)$ matrices $\left[M^{r}\right]$ and $\left[M^{s}\right]$ are defined by

$$
\left[M^{r}\right]=\left[\begin{array}{ccc}
m_{1}\left[I_{3}\right] & \cdots & 0 \\
\vdots & \ddots & \vdots \\
0 & \cdots & m_{n_{b}}\left[I_{3}\right]
\end{array}\right],\left[M^{s}\right]=\left[\begin{array}{ccc}
{\left[J_{1}\right]} & \cdots & 0 \\
\vdots & \ddots & \vdots \\
0 & \cdots & {\left[J_{n_{b}}\right]}
\end{array}\right]
$$

in which $\left[I_{3}\right]$ is the $(3 \times 3)$ identity matrix. The function $\{\mathbf{u}(t), \in[0, T]\}$ is then the solution of the following differential equation (see [15])

$$
\left[\begin{array}{cc}
{[M]} & {\left[\boldsymbol{\varphi}_{\mathbf{u}}\right]^{T}} \\
{\left[\boldsymbol{\varphi}_{\mathbf{u}}\right]} & {[0]}
\end{array}\right]\left[\begin{array}{c}
\ddot{\mathbf{u}} \\
\boldsymbol{\lambda}
\end{array}\right]=\left[\begin{array}{c}
\mathbf{q}-\mathbf{k} \\
-\frac{d}{d t} \boldsymbol{\varphi}_{t}-\left[\frac{d}{d t} \boldsymbol{\varphi}_{\mathbf{u}}\right] \dot{\mathbf{u}}
\end{array}\right]
$$

with the initial conditions

$$
\mathbf{u}(0)=\mathbf{u}_{0} \quad, \quad \dot{\mathbf{u}}(0)=\mathbf{v}_{0} \quad,
$$

in which $\mathbf{k}(\dot{\mathbf{u}})$ is the vector of the Coriolis forces and where $\left[\boldsymbol{\varphi}_{\mathbf{u}}(\mathbf{u}(t), t)\right]_{i j}=\partial \varphi_{i}(\mathbf{u}(t), t) / \partial u_{j}(t)$ and $\varphi_{t}=\partial \varphi / \partial t$. The vector $\mathbf{q}(\mathbf{u}, \dot{\mathbf{u}}, t)$ is constituted of the applied forces and torques induced by springs, dampers and actuators. The vector $\boldsymbol{\lambda}(t)$ is the vector of the Lagrange multipliers. Equation (6) can be solved using an adapted integration algorithm (see for instance [2]). 


\section{STOCHASTIC MODEL FOR A MULTIBODY DYNAMICAL SYSTEM WITH UN- CERTAIN RIGID BODIES}

Firstly, a stochastic model for an uncertain rigid body of the multibody dynamical system is proposed and secondly, the stochastic model for the multibody dynamical system with uncertain rigid bodies is constructed joining the stochastic model of the uncertain rigid bodies.

\subsection{Stochastic model for an uncertain rigid body of the multibody dynamical system}

The properties of the mean model (or the nominal model) of the rigid body $\mathrm{RB}_{i}$ are defined by its mass $\underline{m}_{i}$, the position vector $\underline{\mathbf{r}}_{0, i}$ of its center of mass $\underline{G}_{i}$ at initial time $t=0$ and the matrix $\left[\underline{J}_{i}\right]$ of its tensor of inertia with respect to the local frame $\left(\underline{G}_{i}, \underline{x}_{i, 1}^{\prime}, \underline{x}_{i, 2}^{\prime}, \underline{x}_{i, 3}^{\prime}\right)$. The probabilistic model of uncertainties for this rigid body is constructed by replacing these three parameters by the following three random variables: the random mass $M_{i}$, the random position vector $\mathbf{R}_{0, i}$ of its random center of mass $\mathbf{G}_{i}$ at initial time $t=0$ and the random matrix $\left[\mathbf{J}_{i}\right]$ of its random tensor of inertia with respect to the random local frame $\left(\mathbf{G}_{i}, \underline{x}_{i, 1}^{\prime}, \underline{x}_{i, 2}^{\prime}, \underline{x}_{i, 3}^{\prime}\right)$. The probability density functions (PDF) of these three random variables are constructed using the maximum entropy principle (see [17], [6]), that is to say, in maximizing the uncertainties in the model under the constraints defined by the available information.

\subsubsection{Construction of the PDF for the random mass}

(i) Available information

Let $E\{$.$\} be the mathematical expectation. The available information for the random mass M_{i}$ is defined as follows. Firstly, the random variable $M_{i}$ must be positive almost surely. Secondly, the mean value of the random mass $M_{i}$ must be equal to the value $\underline{m}_{i}$ of the mean (or nominal) model. Thirdly, as it is proven in [19], the random mass must verify the inequality $E\left\{M_{i}^{-2}\right\}<+\infty$ in order that a second-order solution exists for the stochastic dynamical system. In addition, it is also proven that this constraint can be replaced by $\left|E\left\{\log M_{i}\right\}\right|<+\infty$.

(ii) Maximum entropy principle

The probability density function $\mu \mapsto p_{M_{i}}(\mu)$ of the random variable $M_{i}$ is constructed by maximizing the entropy under the constraints defined above. The solution of this optimization problem is the PDF of a gamma random variable defined on $] 0,+\infty[$. This PDF depends on two parameters which are $\underline{m}_{i}$ and $C_{M_{i}}$. Since parameter $C_{M_{i}}$ has no physical meaning, it is eliminated in introducing the coefficient of variation $\delta_{M_{i}}$ of the random variable $M_{i}$ such that $\delta_{M_{i}}=\sigma_{M_{i}} / \underline{m}_{i}$ where $\sigma_{M_{i}}$ is the standard deviation of the random variable $M_{i}$. Therefore, the PDF of the random mass is completely defined by the mean value $\underline{m}_{i}$ and by the dispersion parameter $\delta_{M_{i}}$.

\subsubsection{Construction of the PDF for the random position vector $\mathbf{R}_{0, i}$}

In this subsection, the PDF of the random initial position vector $\mathbf{R}_{0, i}$ of the center of mass of $\mathrm{RB}_{i}$ at initial time $t=0$ is constructed.

\section{(i) Available information}

The position vector $\underline{\mathbf{r}}_{0, i}$ of the center of mass $\underline{G}_{i}$ at initial time $t=0$ of the mean (or nominal) model is given. However, the real position is not exactly known and $\underline{\mathbf{r}}_{0, i}$ only corresponds to a mean position. Consequently, there is an uncertainty about the real position and this is the 
reason why this position is modeled by the random vector $\mathbf{R}_{0, i}$. Some geometrical and mechanical considerations lead us to introduce an admissible domain $D_{i}$ of random vector $\mathbf{R}_{0, i}$. We introduce the vector $\mathbf{h}$ of the parameters describing the geometry of domain $D_{i}$. In addition, the mean value of the random vector $\mathbf{R}_{0, i}$ must be equal to the value $\underline{\mathbf{r}}_{0, i}$ of the mean (or nominal) model. Therefore, the available information for random variable $\mathbf{R}_{0, i}$ can be written as

$$
\begin{gathered}
\mathbf{R}_{0, i} \in D_{i}(\mathbf{h}) \quad \text { a.s. } \quad, \quad(a) \\
E\left\{\mathbf{R}_{0, i}\right\}=\underline{\mathbf{r}}_{0, i} \in D_{i}(\mathbf{h})
\end{gathered}
$$

(ii) Maximum entropy principle

The probability density function $\mathbf{a} \mapsto p_{\mathbf{R}_{0, i}}$ (a) of random variable $\mathbf{R}_{0, i}$ is then constructed by maximizing the entropy with the constraints defined by the available information in Eq. (8). The solution of this optimization problem depends on two parameters which are $\underline{\mathbf{r}}_{0, i}$ and vectorvalued parameter $\mathbf{h}$, and is such that

$$
p_{\mathbf{R}_{0, i}}(\mathbf{a} ; \mathbf{h})=\mathbb{1}_{D_{i}(\mathbf{h})}(\mathbf{a}) C_{0} e^{-<\boldsymbol{\lambda}, \mathbf{a}>} .
$$

The positive valued parameter $C_{0}$ and vector $\boldsymbol{\lambda}$ are the unique solution of the equations

$$
\begin{aligned}
C_{0} \int_{D_{i}(\mathbf{h})} e^{-<\boldsymbol{\lambda}, \mathbf{a}>} d \mathbf{a}=1, \\
C_{0} \int_{D_{i}(\mathbf{h})} \mathbf{a} e^{-<\boldsymbol{\lambda}, \mathbf{a}>} d \mathbf{a}=\underline{\mathbf{r}}_{0, i} .
\end{aligned}
$$

\section{(iii) Generator of independent realizations}

The independent realizations of random variable $\mathbf{R}_{0, i}$ must be generated using the constructed PDF $p_{\mathbf{R}_{0, i}}$. Such a generator can be obtained using the Monte Carlo Markov Chain (MCMC) method (Metropolis-Hastings algorithm [5]).

\subsubsection{Random matrix $\left[J_{i}\right]$ of the random tensor of inertia.}

In this subsection, the random matrix $\left[\mathbf{J}_{i}\right]$ of the random tensor of inertia with respect to $\left(\mathbf{G}_{i}, \underline{x}_{i, 1}^{\prime}, \underline{x}_{i, 2}^{\prime}, \underline{x}_{i, 3}^{\prime}\right)$ is defined and an algebraic representation of this random matrix is constructed. The mass distribution around the random center of mass $\mathbf{G}_{i}$ is uncertain and consequently, the tensor of inertia is also uncertain. This is the reason why the matrix $\left[\underline{J}_{i}\right]$ of the tensor of inertia of the mean (or nominal) model with respect to $\left(\underline{G}_{i}, \underline{x}_{i, 1}^{\prime}, \underline{x}_{i, 2}^{\prime}, \underline{x}_{i, 3}^{\prime}\right)$ is replaced by a random matrix $\left[\mathbf{J}_{i}\right]$ which is constructed by using the maximum entropy principle.

We introduce the positive-definite matrix $\left[Z_{i}\right]$ independent of $m_{i}$ such that

$$
\left[Z_{i}\right]=\frac{1}{m_{i}}\left\{\frac{\operatorname{tr}\left(\left[J_{i}\right]\right)}{2}\left[I_{3}\right]-\left[J_{i}\right]\right\} .
$$

Then $\left[J_{i}\right]$ can be calculated as a function of $\left[Z_{i}\right]$,

$$
\left[J_{i}\right]=m_{i}\left\{\operatorname{tr}\left(\left[Z_{i}\right]\right)\left[I_{3}\right]-\left[Z_{i}\right]\right\} \quad .
$$

It can be proven that $\left[Z_{i}\right]$ is positive definite and that each positive definite matrix $\left[J_{i}\right]$ constructed using Eq. (12), where $\left[Z_{i}\right]$ is a given positive definite matrix, can be interpreted as the matrix of a tensor of inertia of a physical rigid body for which the mass is 1 (see [1]). 
The probabilistic modeling $\left[\mathbf{J}_{i}\right]$ of $\left[J_{i}\right]$ consists in introducing the random matrix $\left[\mathbf{Z}_{i}\right]$ and in using Eq. (12) in which $m_{i}$ is replaced by the random variable $M_{i}$ and where $\left[Z_{i}\right]$ is replaced by $\left[\mathbf{Z}_{i}\right]$. We then obtain

$$
\begin{aligned}
& {\left[\mathbf{Z}_{i}\right]=\frac{1}{M_{i}}\left\{\frac{\operatorname{tr}\left(\left[\mathbf{J}_{i}\right]\right)}{2}\left[I_{3}\right]-\left[\mathbf{J}_{i}\right]\right\},} \\
& {\left[\mathbf{J}_{i}\right]=M_{i}\left\{\operatorname{tr}\left(\left[\mathbf{Z}_{i}\right]\right)\left[I_{3}\right]-\left[\mathbf{Z}_{i}\right]\right\} .}
\end{aligned}
$$

(i) Available information concerning random matrix $\left[\mathbf{Z}_{i}\right]$

Let us introduce (1) the nominal value $\left[\underline{Z}_{i}\right]$ of deterministic matrix $\left[Z_{i}\right]$ such that $\left[\underline{Z}_{i}\right]=$ $\left(1 / \underline{m}_{i}\right)\left\{\operatorname{tr}\left(\left[\underline{J}_{i}\right]\right) / 2\left[I_{3}\right]-\left[\underline{J}_{i}\right]\right\}$ and (2) the upper bound $\left[Z_{i}^{\max }\right]$ of random matrix $\left[\mathbf{Z}_{i}\right]$. Then, the available information for $\left[\mathbf{Z}_{i}\right]$ can be summarized as follows,

$$
\begin{gathered}
{\left[\mathbf{Z}_{i}\right] \in \mathbb{M}_{3}^{+}(\mathbb{R}) \quad \text { a.s. }} \\
\left\{\left[Z_{i}^{\max }\right]-\left[\mathbf{Z}_{i}\right]\right\} \in \mathbb{M}_{3}^{+}(\mathbb{R}) \quad \text { a.s. } \\
E\left\{\left[\mathbf{Z}_{i}\right]\right\}=\left[\underline{Z}_{i}\right] \quad, \\
E\left\{\log \left(\operatorname{det}\left[\mathbf{Z}_{i}\right]\right)\right\}=C_{i}^{l} \quad, \quad\left|C_{i}^{l}\right|<+\infty \quad, \\
E\left\{\log \left(\operatorname{det}\left(\left[Z_{i}^{\max }\right]-\left[\mathbf{Z}_{i}\right]\right)\right)\right\}=C_{i}^{u} \quad, \quad\left|C_{i}^{u}\right|<+\infty \quad .(e)
\end{gathered}
$$

For more convenience, random matrix $\left[\mathbf{Z}_{i}\right]$ is normalized as follow. Matrix $\left[\underline{Z}_{i}\right]$ being positive definite, its Cholesky decomposition yields $\left[\underline{Z}_{i}\right]=\left[\underline{L}_{Z_{i}}\right]^{T}\left[\underline{L}_{Z_{i}}\right]$ in which $\left[\underline{L}_{Z_{i}}\right]$ is an upper triangular matrix in the set $\mathbb{M}_{3}(\mathbb{R})$ of all the $(3 \times 3)$ real matrices. Then, random matrix $\left[\mathbf{Z}_{i}\right]$ can be rewritten as

$$
\left[\mathbf{Z}_{i}\right]=\left[\underline{L}_{Z_{i}}\right]^{T}\left[\mathbf{G}_{i}\right]\left[\underline{L}_{Z_{i}}\right],
$$

in which the matrix $\left[\mathbf{G}_{i}\right]$ is a random matrix for which the available information is

$$
\begin{gathered}
{\left[\mathbf{G}_{i}\right] \in \mathbb{M}_{3}^{+}(\mathbb{R}) \quad \text { a.s. }} \\
\left\{\left[G_{i}^{\max }\right]-\left[\mathbf{G}_{i}\right]\right\} \in \mathbb{M}_{3}^{+}(\mathbb{R}) \quad \text { a.s. }, \\
E\left\{\left[\mathbf{G}_{i}\right]\right\}=\left[I_{3}\right], \\
E\left\{\log \left(\operatorname{det}\left[\mathbf{G}_{i}\right]\right)\right\}=C_{i}^{l^{\prime}}, \quad\left|C_{i}^{l^{\prime}}\right|<+\infty, \\
E\left\{\log \left(\operatorname{det}\left(\left[G_{i}^{\max }\right]-\left[\mathbf{G}_{i}\right]\right)\right)\right\}=C_{i}^{u^{\prime}} \quad, \quad\left|C_{i}^{u^{\prime}}\right|<+\infty,
\end{gathered}
$$

in which $C_{i}^{l^{\prime}}=C_{i}^{l}-\log \left(\operatorname{det}\left[\underline{Z}_{i}\right]\right), C_{i}^{u^{\prime}}=C_{i}^{u}-\log \left(\operatorname{det}\left[\underline{Z}_{i}\right]\right)$ and where the matrix $\left[G_{i}^{\text {max }}\right]$ is an upper bound for random matrix $\left[\mathbf{G}_{i}\right]$ and is defined by $\left[G_{i}^{\max }\right]=\left(\left[\underline{L}_{Z_{i}}\right]^{T}\right)^{-1}\left[Z_{i}^{\max }\right]\left[\underline{L}_{Z_{i}}\right]^{-1}$.

(ii) Maximum entropy principle

The probability distribution of random matrix $\left[\mathbf{G}_{i}\right]$ is constructed using the maximum entropy principle under the constraints defined by the available information given by Eq. (17). The probability density function $p_{\left[\mathbf{G}_{i}\right]}([G])$ with respect to the volume element $\widetilde{d} G$ of random matrix $\left[\mathbf{G}_{i}\right]$ is then written as

$$
\begin{aligned}
& p_{\left[\mathbf{G}_{i}\right]}([G])=\mathbb{1}_{\mathbb{M}_{3}^{+}(\mathbb{R})}([G]) \times \mathbb{1}_{\mathbb{M}_{3}^{+}(\mathbb{R})}\left(\left[G_{i}^{\text {max }}\right]-[G]\right) \times C_{G_{i}} \\
& \times(\operatorname{det}[G])^{-\lambda_{l}} \times\left(\operatorname{det}\left(\left[G_{i}^{\text {max }}\right]-[G]\right)\right)^{-\lambda_{u}} \times e^{-\operatorname{tr}([\mu][G])},
\end{aligned}
$$


in which the positive valued parameter $C_{G_{i}}$ is a normalization constant, the real parameters $\lambda_{l}<1$ and $\lambda_{u}<1$ are Lagrange multipliers relative to the two last constraints defined by Eq. (17) and the symmetric real matrix $[\mu]$ is a Lagrange multiplier relative to the third constraint defined by Eq. (17). This probability density function is a particular case the Kummer-Beta matrix variate distribution (see [11], [4]) for which the lower bound is a zero matrix.

Parameters $C_{G_{i}}, \lambda_{l}, \lambda_{u}$ and matrix $[\mu]$ are the unique solution of the equations

$$
\begin{gathered}
E\left\{\mathbb{1}_{\mathbb{M}_{3}^{S}(\mathbb{R})}\left(\left[\mathbf{G}_{i}\right]\right)\right\}=1, \\
E\left\{\left[\mathbf{G}_{i}\right]\right\}=\left[I_{3}\right], \\
E\left\{\log \left(\operatorname{det}\left[\mathbf{G}_{i}\right]\right)\right\}=C_{i}^{\prime \prime}, \\
E\left\{\log \left(\operatorname{det}\left(\left[G_{i}^{\max }\right]-\left[\mathbf{G}_{i}\right]\right)\right)\right\}=C_{i}^{u \prime} .
\end{gathered}
$$

For fixed values of $\lambda_{l}$ and $\lambda_{u}$, parameters $C_{G_{i}}$ and $[\mu]$ can be estimated using Eq. (19). In Eq. (19), since the parameters $C_{i}^{l^{\prime}}$ and $C_{i}^{u \prime}$ have no real physical meaning, the parameters $\lambda_{l}$ and $\lambda_{u}$ are kept as parameters which then allows the "shape" of the PDF to be controlled. If experimental data are available for the responses of the dynamical system, then the two parameters $\lambda_{l}$ and $\lambda_{u}$ can be identified solving an inverse problem. If experimental data are not available, these two parameters allow a sensitivity analysis of the solution to be carried out with respect to the level of uncertainties.

(iii) Properties for random matrix $\left[\boldsymbol{J}_{i}\right]$

It is proven in [1] that using Eq. (14) and the available information defined by Eq. (15), the following important properties for random matrix $\left[\mathbf{J}_{i}\right]$ can be deduced,

$$
\begin{gathered}
\left\{\frac{1}{2} \operatorname{tr}\left(\left[\mathbf{J}_{i}\right]\right)\left[I_{3}\right]-\left[\mathbf{J}_{i}\right]\right\} \in \mathbb{M}_{3}^{+}(\mathbb{R}) \quad \text { a.s }, \\
\left\{\left[\mathbf{J}_{i}^{\text {max }}\right]-\left[\mathbf{J}_{i}\right]\right\} \in \mathbb{M}_{3}^{+}(\mathbb{R}) \quad \text { a.s }, \\
E\left\{\left[\mathbf{J}_{i}\right]\right\}=\left[\underline{J}_{i}\right], \\
\left\{\lambda_{l}<-2, \lambda_{u}<0\right\} \Rightarrow E\left\{\left\|\left[\mathbf{J}_{i}\right]^{-1}\right\|^{2}\right\}<+\infty,
\end{gathered}
$$

in which the random matrix $\left[\mathbf{J}_{i}^{\max }\right]$, which represents a random upper bound for random matrix $\left[\mathbf{J}_{i}\right]$, is defined by

$$
\left[\mathbf{J}_{i}^{\max }\right]=M_{i}\left\{\operatorname{tr}\left(\left[Z_{i}^{\max }\right]\right)\left[I_{3}\right]-\left[Z_{i}^{\max }\right]\right\}
$$

It should be noted that Eq. (20-a) implies that each realization of random matrix $\left[\mathbf{J}_{i}\right]$ corresponds to the matrix of a tensor of inertia of a physical rigid body. In addition, this equation implies that random matrix $\left[\mathbf{J}_{i}\right]$ is almost surely positive definite. Eq. $(20-\mathrm{b})$ provides a random upper bound for random matrix $\left[\mathbf{J}_{i}\right]$. Eq. (20-c) corresponds to a construction for which the mean value of random matrix $\left[\mathbf{J}_{i}\right]$ is equal to the nominal value $\left[\underline{J}_{i}\right]$. Finally, Eq. $(20$-d) is necessary for that the random solution of the nonlinear dynamical system be a second-order stochastic process.

\section{(iv) Generator of independent realizations for random matrix $\left[\boldsymbol{J}_{i}\right]$}

The generator of independent realizations of random matrix $\left[\mathbf{G}_{i}\right]$ is based on the Monte Carlo Markov Chain (MCMC) (Metropolis-Hastings algorithm [5] with the PDF defined by Eq. (18). Then, independent realizations of random matrix $\left[\mathbf{Z}_{i}\right]$ are obtained using Eq. (16). Finally, independent realizations of random matrix $\left[\mathbf{J}_{i}\right]$ are obtained using Eq. (14) and independent realizations of random mass $M_{i}$. 


\subsection{Stochastic matrix model for a multibody dynamical system with uncertain rigid bod- ies and its random response}

In order to limit the developments, it is assumed that only one of the $n_{b}$ rigid bodies denoted by $\mathrm{RB}_{i}$ of the rigid multibody system is uncertain. The extension to several uncertain rigid bodies is straightforward. Let the $6 n_{b}$ random coordinates be represented by the $\mathbb{R}^{6 n_{b}}$ valued stochastic process $\mathbf{U}=\left(\mathbf{R}_{1}, \ldots, \mathbf{R}_{n_{b}}, \mathbf{S}_{1}, \ldots, \mathbf{S}_{n_{b}}\right)$ indexed by $[0, T]$ and let the $n_{c}$ random

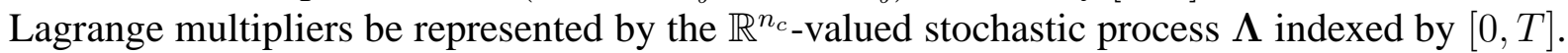
The deterministic Eq. (6) becomes the following stochastic equation

$$
\begin{gathered}
{\left[\begin{array}{cc}
{[\mathbf{M}]} & {\left[\boldsymbol{\varphi}_{\mathbf{u}}\right]^{T}} \\
{\left[\boldsymbol{\varphi}_{\mathbf{u}}\right]} & {[0]}
\end{array}\right]\left[\begin{array}{c}
\ddot{\mathbf{U}} \\
\boldsymbol{\Lambda}
\end{array}\right]=\left[\begin{array}{c}
\mathbf{q}-\mathbf{K} \\
-\frac{d}{d t} \boldsymbol{\varphi}_{t}-\left[\frac{d}{d t} \boldsymbol{\varphi}_{\mathbf{u}}\right] \dot{\mathbf{U}}
\end{array}\right],} \\
\mathbf{U}(0)=\mathbf{U}_{0} \quad, \quad \dot{\mathbf{U}}(0)=\mathbf{v}_{0} \quad, \quad \text { a.s. }
\end{gathered}
$$

in which the vector $\mathbf{U}_{0}=\left(\mathbf{r}_{0,1}, \ldots, \mathbf{R}_{0, i}, \ldots, \mathbf{r}_{0, n_{b}}, \mathbf{s}_{0,1}, \ldots, \mathbf{s}_{0, n_{b}}\right)$ is random due to the random vector $\mathbf{R}_{0, i}$. For all given real vector $\dot{\mathbf{u}}$, the vector $\mathbf{K}(\dot{\mathbf{u}})$ of the Coriolis forces is random due to the random matrix $\left[\mathbf{J}_{i}\right]$. The random mass matrix $[\mathbf{M}]$ is defined by

$$
[\mathbf{M}]=\left[\begin{array}{cc}
{\left[\mathbf{M}^{r}\right]} & 0 \\
0 & {\left[\mathbf{M}^{s}\right]}
\end{array}\right],
$$

in which the $\left(3 n_{b} \times 3 n_{b}\right)$ random matrices $\left[\mathbf{M}^{r}\right]$ and $[\mathbf{M}]^{s}$ are defined by

$$
\begin{aligned}
& {\left[\mathbf{M}^{r}\right]=\left[\begin{array}{ccccc}
m_{1}\left[I_{3}\right] & & \ldots & & 0 \\
\vdots & \ddots & & & \\
& & \ldots & \ddots & m_{n_{b}}\left[I_{3}\right] \\
0 & & \ldots & &
\end{array}\right]} \\
& {\left[\mathbf{M}^{s}\right]=\left[\begin{array}{ccccc}
{\left[J_{1}\right]} & & \ldots & & 0 \\
\vdots & \ddots & & & \\
0 & & \ldots & \ddots & {\left[J_{i}\right]} \\
& & \vdots
\end{array}\right]}
\end{aligned}
$$

Random Eqs. (22) and (23) are solved using the Monte Carlo simulation method.

\section{APPLICATION}

In this section, we present a numerical application which validates the methodology presented in this paper.

\subsection{Description of the mean model}

The rigid multibody model is made up of five rigid bodies and six joints which are described in the fixed frame $\left(O, x_{0,1}, x_{0,2}, x_{0,3}\right)$ (see Fig. 1). The plan defined by $\left(O, x_{0,1}, x_{0,2}\right)$ is identified below as the "ground". The gravity forces in the $x_{0,3}$-direction are taken into account.

(i) Rigid bodies

In the initial configuration, the rigid bodies $R b 1, R b 2, R b 3$ and $R b 4$ are cylinders for which the 


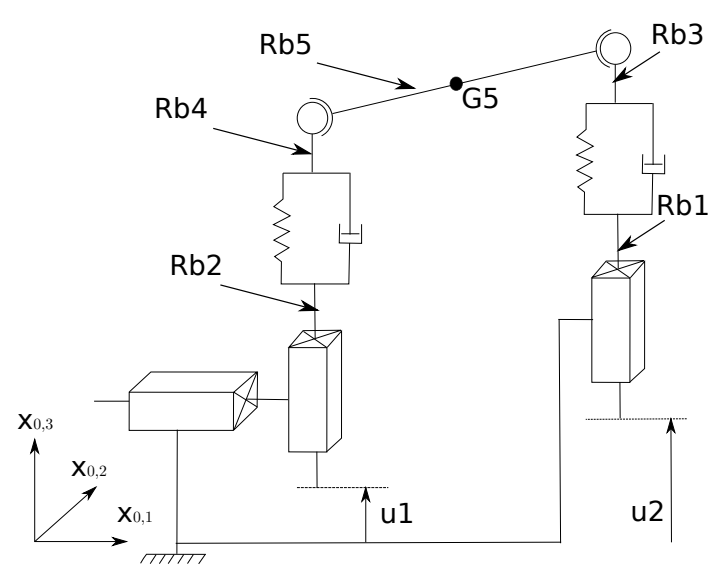

Figure 1: Rigid multibody system.
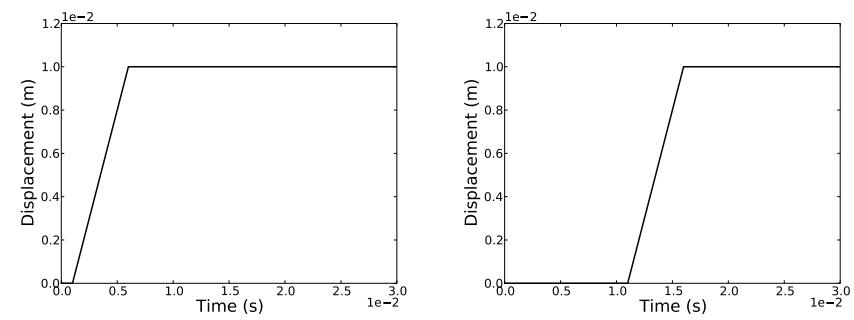

Figure 2: Imposed displacement $u 1(t)$ (left figure) and $u 2(t)$ (right figure).

axes follow the $x_{0,3}$-direction. In the initial configuration, the rigid body $R b 5$ is supposed to be symmetric with respect to the planes $\left(G 5, x_{0,1}, x_{0,2}\right)$ and $\left(G 5, x_{0,1}, x_{0,3}\right)$ in which $G 5$ is the center of mass of $R b 5$.

(ii) Joints

- The joint Ground-Rb1 is made up of a prismatic joint following $x_{0,3}$-direction. The displacement following $x_{0,3}$-direction (see Fig. 1 ), denoted by $u 1(t)$, is imposed. The joint Ground-Rb2 is a prismatic joint following $x_{0,3}$-direction. The displacement following $x_{0,3}$-direction (see Fig. 11), denoted by $u 2(t)$, is imposed. The displacement following $x_{0,1}$-direction is unconstrained. Imposed displacements $u 1(t)$ and $u 2(t)$ are plotted in Fig. 2 for $t$ in $[0,0.03] s$.

- The joints $R b 1-R b 3$ and $R b 2-R b 4$ are constituted of $6 \mathrm{D}$ spring-dampers..

- Finally, the joints $R b 3-R b 5$ and $R b 4-R b 5$ are $x_{0,2}$-direction revolute joints.

\subsection{Random response of the stochastic model}

Rigid body $R b 5 a$ is considered as uncertain and is therefore modeled by a random rigid body. As explained in Section 3, the elements of inertia of the uncertain rigid Body $R b 5$ are replaced by random quantities. The fluctuation of the response is controlled by four parameters $\delta_{M_{5}}, \mathbf{h}$, $\lambda_{l}$ and $\lambda_{u}$. A sensitivity analysis is carried out with respect to these four parameters. Statistics on the transient response are estimated using the Monte Carlo simulation method with 500 independent realizations. The initial velocities and angular velocities are zero. The observation point $P_{o b s}$ belongs to $R b 5$.

(i) Case 1: $M_{5}$ is random, $\mathbf{R}_{0,5}$ is deterministic and $\left[\mathbf{J}_{5}\right]$ is deterministic.

We choose $\delta_{M_{5}}=0.5$. The confidence region, with a probability level $P_{c}=0.90$, of the random 

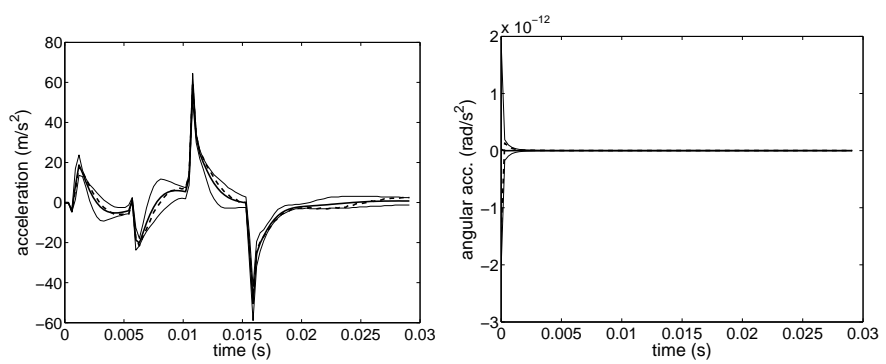

Figure 3: Random transient acceleration of point $P_{o b s}$, Case 1: confidence region (upper and lower thin solid lines), mean response (thick solid line) and response of the mean model (dashed line); $x_{0,3}$-acceleration (left figure) and $x_{0,1}$-angular acceleration (right figure).
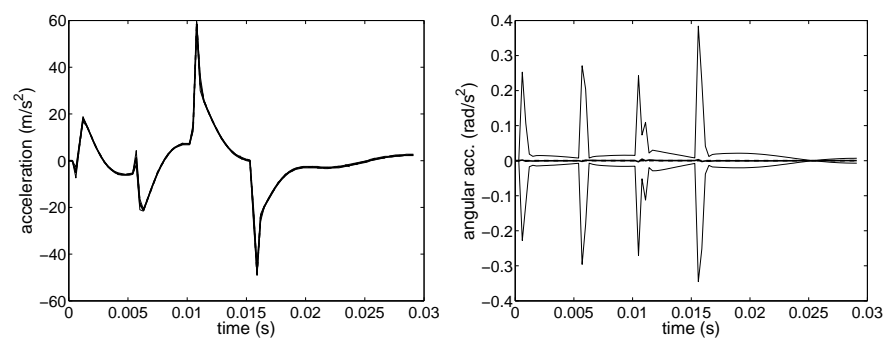

Figure 4: Random transient acceleration of point $P_{o b s}$, Case 2: confidence region (upper and lower envelopes), mean response and response of the mean model are superimposed; $x_{0,3}$-acceleration (left figure) and $x_{0,1}$-angular acceleration (right figure).

acceleration of point $P_{\text {obs }}$ is plotted in Fig. 3. It can be noted that the acceleration is sensitive to the mass uncertainties.

(ii) Case 2: $M_{i}$ is deterministic, $\mathbf{R}_{0,5}$ is deterministic and $\left[\mathbf{J}_{5}\right]$ is random.

We choose $\lambda_{l}=-5$ and $\lambda_{u}=-5$ for random matrix $\left[\mathbf{J}_{5}\right]$. The confidence region, with a probability level $P_{c}=0.90$, of the random acceleration of point $P_{o b s}$ is plotted in Fig. 4. We can remark, as it was expected, that the angular acceleration is sensitive to uncertainties on the tensor of inertia.

(iii) Case 3: $M_{5}$ is deterministic, $\mathbf{R}_{0,5}$ is random and $\left[\mathbf{J}_{5}\right]$ is deterministic.

The domain of $\mathbf{R}_{0,5}$ is supposed to be a parallelepiped which is centered at point $(0,0,0.55)$ for which its edges are parallel to the directions $x_{0,1}, x_{0,2}$ and $x_{0,3}$ and for which the lengths following these three directions are respectively $0.5,0.2$ and 0.02 . The confidence region, with a probability level $P_{c}=0.90$, of the random acceleration of point $P_{o b s}$ is plotted in Fig. 5. We can remark that the angular acceleration is sensitive to uncertainties on initial center of mass of $R b 5$.

(iv) Case 4: $M_{5}, \mathbf{R}_{0,5}$ and $\left[\mathbf{J}_{5}\right]$ are random.

The values of the parameters of the PDF are those fixed in the three previous cases. The confidence region, with a probability level $P_{c}=0.90$, of the random acceleration of point $P_{\text {obs }}$ is plotted in Fig. 6. It can be viewed that (1) the randomness on the acceleration is mainly due to the randomness of mass $M_{5}$, (2) the randomness on the angular acceleration is mainly due to the randomness of the initial position $\mathbf{R}_{0,5}$ of the center of mass and the random tensor of inertia $\left[\mathbf{J}_{5}\right]$. 

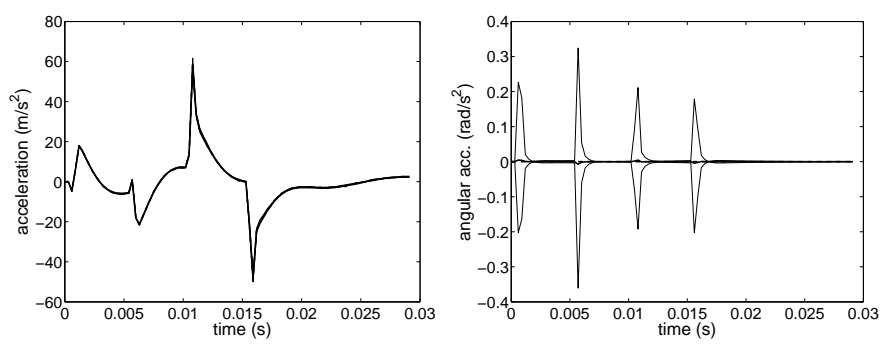

Figure 5: Random transient acceleration of point $P_{o b s}$, Case 3: confidence region (upper and lower envelopes), mean response and response of the mean model are superimposed; $x_{0,3}$-acceleration (left figure) and $x_{0,1}$-angular acceleration (right figure).
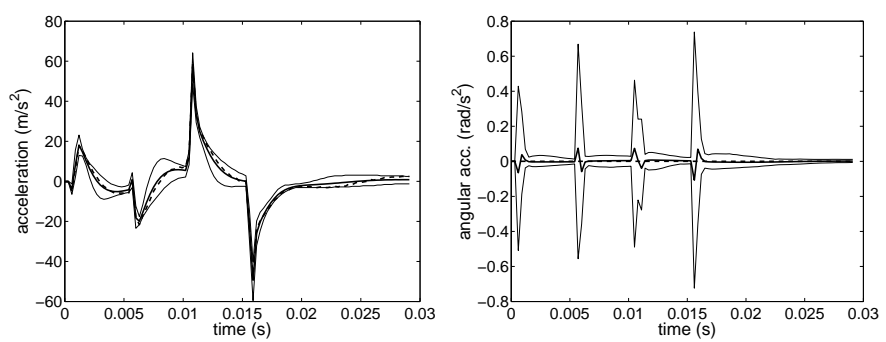

Figure 6: Random transient acceleration of point $P_{o b s}$, Case 4: confidence region (upper and lower thin solid lines), mean response (thick solid line) and response of the mean model (dashed line); $x_{0,3}$-acceleration (left figure) and $x_{0,1}$-angular acceleration (right figure).

\section{CONCLUSION}

We have presented a complete and general probabilistic modeling of uncertain rigid bodies taking into account all the known mechanical and mathematical properties. This probabilistic model of uncertainties is used to construct the stochastic equations of uncertain multibody dynamical systems. The random dynamical responses can then be calculated. In the proposed probabilistic model, the mass, the center of mass and the tensor of inertia are modeled by random variables for which the prior probability density functions are constructed using the maximum entropy principle under the constraints defined by all the available mathematical, mechanical and design properties. Several uncertain rigid bodies can be linked each others in order to obtain the stochastic dynamical model of the uncertain multibody dynamical system. The theory proposed has been illustrated analyzing a simple example. The results obtained clearly show the role played by uncertainties and the sensitivity of the responses due to uncertainties on (1) the mass (2) the center of mass and (3) the tensor of inertia. Such a prior stochastic model allows the robustness of the responses to be analyzed with respect to uncertainties. If experimental data were available on the responses, then the parameters which control the level of uncertainties could be estimated by solving an inverse stochastic problem.

\section{REFERENCES}

[1] A. Batou, C. Soize, Rigid multibody system dynamics with uncertain rigid bodies, Multibody System Dynamics. submitted in 2010.

[2] J. Baumgarte, Stabilization of constraints and integrals of motion in dynamical systems, Computer Methods in Applied Mechanics and Engineering. 1(1), 1-16, 1972. 
[3] A. Carrarini, Reliability based analysis of the crosswind stability of railway vehicles, Journal of Wind Engineering and Industrial Aerodynamics. 95, 493-509, 2007.

[4] S. Das, R. Ghanem, A bounded random matrix approach for stochastic upscaling, Multiscale Modeling \& Simulation. 8(1), 296-325, 2009.

[5] W.K. Hastings, Monte Carlo sampling methods using Markov chains and their applications, Biometrika. 109, 57-97, 1970.

[6] E.T. Jaynes, Information theory and statistical mechanics, Physical Review. 106(4), 620630 and 108(2), 171-190, 1957.

[7] L. Li, C. Corina Sandu, On the impact of cargo weight, vehicle parameters, and terrain characteristics on the prediction of traction for off-road vehicles, Journal of Terramechanics. 44, 221-238, 2007.

[8] P. Masarati, M. Lanz, P. Mantegazza, Multistep integration of ordinary, stiff and differential-algebraic problems for multibody dynamics applications, $16^{\text {th }}$ Congresso Nazionale AIDAA. 24-28 Septembre, Palermo, Italy, 2001.

[9] R. Murthy, M.P. Mignolet, A. El-Shafei, Nonparametric stochastic modeling of uncertainty in rotordynamics-Part I: Formulation, J. Eng. Gas Turbines Power. 132(9), 092501, 2010.

[10] R. Murthy, M.P. Mignolet, A. El-Shafei, Nonparametric stochastic modeling of uncertainty in rotordynamics-Part II: Applications, J. Eng. Gas Turbines Power. 132(9), 092502, 2010.

[11] D.K Nagar, A.K. Gupta, Matrix-variate Kummer-Beta distribution, Journal of the Australian Mathematical Society. 73, 11-25, 2002.

[12] D. Negrut, M. Datar, D. Gorsich., D. Lamb , A framework for uncertainty quantification in nonlinear multi-body system dynamics, Proceedings of the 26th Army Science Conference. Orlando, FL, 2008.

[13] A. Sandu, C. Sandu, M. Ahmadian, Modeling multibody dynamic systems with uncertainties. Part I: theoretical and computational aspects, Multibody System Dynamics, 23, 375-395, 2006.

[14] W. Schiehlen, Multibody Systems Handbook. Springer-Verlag, Berlin, 1990.

[15] W. Schiehlen, Multibody system dynamics: roots and perspectives, Multibody System Dynamics. 1, 149-188, 1997.

[16] K.P. Schmitt, M. Anitescu, D. Negrut, Efficient sampling for spatial uncertainty quantification in multibody system dynamics applications, Int. J. Numer. Meth. Engng. 80, 537-564, 2009.

[17] C.E. Shannon, A mathematical theory of communication, Bell System Technology Journal. 27, 379-423 and 623-659, 1948. 
[18] C. Soize, A nonparametric model of random uncertainties on reduced matrix model in structural dynamics, Probabilistic Engineering Mechanics. 15(3), 277-294, 2000.

[19] C. Soize, Maximum entropy approach for modeling random uncertainties in transient elastodynamics, J. Acoust. Soc. Am. 109(5), 1979-1996, 2001. 